DESY 10-058

IPMU 10-0075

\title{
Dynamical Matter-Parity Breaking and Gravitino Dark Matter
}

\author{
Jonas Schmidt ${ }^{1,2}$, Christoph Weniger ${ }^{1,2}$, Tsutomu T. Yanagida ${ }^{2,3}$ \\ 1 Deutsches Elektronen-Synchrotron (DESY), Notkestrasse 85, 22603 Hamburg, Germany \\ 2 Institute for the Physics and Mathematics of the Universe (IPMU), University of Tokyo, \\ Chiba 277-8568, Japan \\ 3 Department of Physics, University of Tokyo, Tokyo 113-0033, Japan
}

\begin{abstract}
Scenarios where gravitinos with $\mathrm{GeV}$ masses make up dark matter are known to be in tension with high reheating temperatures, as required by e.g. thermal leptogenesis. This tension comes from the longevity of the NLSPs, which can destroy the successful predictions of the standard primordial nucleosynthesis. However, a small violation of matter parity can open new decay channels for the NLSP, avoiding the BBN problems, while being compatible with experimental cosmic-ray constraints. In this paper, we propose a model where matter parity, which we assume to be embedded in the $\mathrm{U}(1)_{B-L}$ gauge symmetry, is broken dynamically in a hidden sector at low scales. This can naturally explain the smallness of the matter parity breaking in the visible sector. We discuss the dynamics of the corresponding pseudo Nambu-Goldstone modes of $B-L$ breaking in the hidden sector, and we comment on typical cosmic-ray and collider signatures in our model.
\end{abstract}




\section{Introduction}

Rapid proton decay in supersymmetric extensions of the standard model is usually avoided by the introduction of $R$ - (or matter-) parity. However, it turns out that in scenarios with gravitino dark matter with masses in the range 10 to a few $100 \mathrm{GeV}$, a small violation of this symmetry can be desirable since this would reconcile thermal leptogenesis as a source for the baryon asymmetry in the universe with standard primordial baryogenesis [1] (for a recent exhaustive study of small bilinear $R$-parity violation see [2]). The tight upper bounds on the corresponding parity-violating couplings, stemming from laboratory experiments [3, 4] and the non-observation of gamma-ray lines from gravitino decays at Fermi LAT [5], then raise the question how the presence of such small couplings can be understood theoretically. In this paper we consider the possibility that the small violation of matter parity, which we assume to be embedded into the $\mathrm{U}(1)_{B-L}$ gauge symmetry, is linked to the condensation of strongly interacting hidden sector fields at low energies.

Thermal leptogenesis [6] via the out-of-equilibrium decay of heavy right-handed neutrinos is one of the most promising models for the generation of the baryon asymmetry in the universe. Furthermore, beside explaining the observed excess of baryons over anti-baryons, the introduction of right-handed neutrinos can also naturally explain the smallness of the neutrino masses; this is known as the see-saw mechanism [7, 8]. Leptogenesis as well as the see-saw mechanism favor very high masses for the right-handed neutrinos, and it is well known that especially thermal leptogenesis requires that the mass of the lightest righthanded neutrino should be larger than $10^{9} \mathrm{GeV}$ [9], which gives also a strong lower limit on the reheating temperature.

In supersymmetric extensions of the standard model, gravitinos play a distinct role due to their Planck-suppressed couplings to the observable world, and they are an excellent candidate for being the dark matter observed in the universe. It is well known that, given a high reheating temperature $T_{R} \sim \mathcal{O}\left(10^{9}-10^{10} \mathrm{GeV}\right)$ and gravitino masses around $m_{3 / 2} \sim \mathcal{O}(10-100 \mathrm{GeV})$, the thermal relic density of gravitinos reproduces the correct dark matter abundance [10], in good agreement with thermal leptogenesis.

However, the above scenario is not free of problems: The next-to-lightest supersymmetric particle (NLSP), which is often the stau, typically decays into the gravitino with lifetimes that are of the order of minutes or days. This is generically in conflict with the successful predictions of standard primordial nucleosynthesis (BBN), since the NLSP decay releases large amounts of electromagnetic or hadronic energy into the primordial plasma, destroying some of the fragile elements and changing their abundance [11,12]. Furthermore, if the NLSP is charged, it could form bound states with ${ }^{4} \mathrm{He}$ and ${ }^{8} \mathrm{Be}$, leading to a catalytic over-production of ${ }^{6} \mathrm{Li}$ and ${ }^{9} \mathrm{Be}$ [13, 14.

Different solutions to the above problem were proposed: If in some special scenarios the sneutrino or the stop is the NLSP, their late decay would not affect the predictions of BBN strongly [15, 16]. In case of stau NLSPs, a large left-right mixing could lead to suppressed relic abundances [17]. Entropy production between thermal freeze-out of the NLSP and BBN could dilute the NLSP and gravitino abundance enough to evade the BBN constraints [18, 19]. The NLSP mass could be nearly degenerate with the gravitino 
mass, which reduces the released energy [20. Or the NLSP could decay into additional hidden sector states before conflicting with BBN [21,22]. Lastly, as mentioned above, in cases where $R$-parity is weakly violated, the NLSP can decay into standard model particles before the onset of BBN [1].

Theoretically, the existence of $R$-parity is not automatic. If it is a global symmetry it is expected to suffer from quantum gravitational 'wormhole' effects [23, 24]. Therefore, it is necessary to assume an embedding into a local symmetry, which is spontaneously broken at a high scale. However, the requirement of anomaly cancellation strongly disfavors gauged $\mathrm{U}(1)_{R}$ proposals. Another possibility is to understand discrete $R$-symmetry [25] in the framework of higher-dimensional constructions. The gauging then corresponds to local coordinate rotations in the extra dimensions, and the $R$-parity of the effective theory is a relic of the higher-dimensional Lorentz group. In string theory such discrete $R$-symmetries may appear in specific compactifications [26], however, not generically.

Alternatively, proton stability in supersymmetric setups can be guaranteed by matter parity. This is natural in models which incorporate the see-saw mechanism, since the required Majorana mass $M_{S}$ of the right-handed neutrinos violates $B-L$ by two units, thereby breaking $\mathrm{U}(1)_{B-L}$ to its matter parity subgroup. Note that $\mathrm{U}(1)_{B-L}$ is the only anomaly-free Abelian extension of the standard model, and that it is canonically included in SO(10) GUT proposals. Therefore, we consider the 'matter parity' to be the $\mathbb{Z}_{2}$ subgroup of the anomaly-free $\mathrm{U}(1)_{B-L}$ gauge symmetry throughout this paper.

As argued above, the violation of $R$-parity or matter parity is bound to be very small. This can be achieved by e.g. connecting $R$-parity breaking with $B-L$ breaking, via nonrenormalizable operators [1] or at loop level in the context of string theory [27].

In this paper, we discuss a scenario where the small matter-parity breaking is related to the condensation scale $\Lambda$ of an asymptotically free hidden sector gauge group factor. This scale can be naturally much lower than the $\mathrm{U}(1)_{B-L}$ breaking scale $M_{S}$. If the hidden sector is charged under $B-L$, the condensate breaks matter parity at low scale. It then dominantely couples to the right-handed neutrinos, thereby inducing a sneutrino vacuum expectation value and consequently small bilinear matter parity violation [28] of the order $\Lambda^{2} / M_{S}$, which can lie in the phenomenologically preferred regime.

The paper is organized as follows: In the next section we will introduce the hidden sector model and discuss its coupling to the visible sector; in section 3 we will discuss phenomenological aspects of the model, namely gravitino LSP and stau NLSP decay, ther-

mal production of hidden sector particles and their decay; section 4 is devoted to further discussions and in section 5 we will conclude.

\section{$2 \quad$ A Model for Dynamical Matter-Parity Breaking}

We consider an extension of the supersymmetric standard model with gauge group

$$
G=\mathrm{SU}(3)_{c} \times \mathrm{SU}(2)_{L} \times \mathrm{U}(1)_{Y} \times \mathrm{U}(1)_{B-L} \times \mathrm{SU}(2)_{\text {hid }}
$$


where $\mathrm{U}(1)_{B-L}$ will be broken spontaneously to matter parity at the high scale $M_{S}$, and $\mathrm{SU}(2)_{\text {hid }}$ is the gauge group in the hidden sector, responsible for the low-energy matterparity breaking.

We assume that light neutrino masses are generated in the standard way by the see-saw mechanism after the breaking of $\mathrm{U}(1)_{B-L}$ at a high scale $M_{S}$. Large Majorana masses for the right-handed neutrinos $N^{c}$ are generated by a singlet field $S$ in our model,

$$
W_{\text {see-saw }}=h_{i j}^{(n)} L_{i} N_{j}^{c} H_{u}+\frac{1}{2} \lambda_{i}^{S} S N_{i}^{c} N_{i}^{c},
$$

where we have taken the basis where the Majorana mass matrix for $N^{c}$ is diagonal. We assume $\langle S\rangle=M_{S}$. Then, the $N_{i}^{c}$ have Majorana masses, $M_{i}=\lambda_{i}^{S} \times M_{S}$ for $i=1-3$, respectively, and we define $M_{1}<M_{2}<M_{3}$ (we will assume that $M_{3} \simeq M_{S}$ below). For successful thermal leptogenesis, we require $M_{1}>10^{9} \mathrm{GeV}$. Since $\mathrm{U}(1)_{B-L}$ is gauged initially, the corresponding Nambu-Goldstone (NG) mode will be absorbed by the gauge field, which then decouples from the low-energy effective theory. The field $S$ necessarily has $B-L$ charge -2 , hence a global discrete $\mathbb{Z}_{2}$ subgroup remains unbroken during this process 1 , the 'matter parity'. We also assume the presence of a field $\bar{S}$ with $B-L$ charge +2 , required for anomaly cancellation, without discussing the dynamics that give rise to the condensation of $S$ and $\bar{S}$ in this paper.

The remaining matter-parity is then broken dynamically by the condensation of quarks in the gauge group $\mathrm{SU}(2)_{\text {hid }}$ at the low scale $\Lambda \ll M_{S}$. The hidden sector contains two doublet quarks $\mathcal{Q}_{1}^{\alpha}, \mathcal{Q}_{2}^{\alpha}$ with $B-L$ charge $+1 / 2$, and two doublet quarks $\mathcal{Q}_{3}^{\alpha}, \mathcal{Q}_{4}^{\alpha}$ with charge $-1 / 2$. Furthermore, we require the existence of five neutral singlets $Z_{13}, Z_{14}, Z_{23}, Z_{24}$ and $X$. Here and in the following $\alpha, \beta=1,2$ are $\mathrm{SU}(2)_{\text {hid }}$ indices. The low-energy degrees of freedom are the antisymmetric combinations [29]

$$
V_{i j}=-V_{j i}=\frac{1}{\Lambda} \mathcal{Q}_{i}^{\alpha} \mathcal{Q}_{j \alpha}
$$

with convention $\mathcal{Q}_{i \alpha}=\epsilon_{\alpha \beta} \mathcal{Q}_{i}^{\beta}$, where $\epsilon_{\alpha \beta}$ is totally antisymmetric and $\epsilon_{12}=1$.

We consider the effective superpotential (cf. [29,30])

$$
W_{\mathrm{dyn}}=X\left(\operatorname{Pf}\left(V_{i j}\right)-\Lambda^{2}\right)+\Lambda\left(Z_{13} V_{13}+Z_{14} V_{14}+Z_{23} V_{23}+Z_{24} V_{24}\right),
$$

where $\operatorname{Pf}\left(V_{i j}\right)=V_{12} V_{34}+V_{14} V_{23}-V_{13} V_{24}$ is the Pfaffian of the antisymmetric matrix $V_{i j}$ and the $X$ is some composite state. The accidental symmetries of the model will be discussed in Section 4 .

The superpotential (2.4) possesses a unique supersymmetric minimum (which is in contrast to [30]), with non-vanishing vacuum expectation values for the two charged effective mesons,

$$
\begin{gathered}
\left\langle V_{13}\right\rangle=\left\langle V_{14}\right\rangle=\left\langle V_{23}\right\rangle=\left\langle V_{24}\right\rangle=0, \\
\left\langle V_{12}\right\rangle=\left\langle V_{34}\right\rangle=\Lambda, \quad\left\langle Z_{14}\right\rangle=\left\langle Z_{23}\right\rangle=\left\langle Z_{24}\right\rangle=\langle X\rangle=0 .
\end{gathered}
$$

\footnotetext{
${ }^{1}$ The generator of matter parity combines $B-L$ with hypercharge, $P=(-)^{B-L+4 Y}$.
} 
Here, we assumed that $\left\langle V_{12}\right\rangle=\left\langle V_{34}\right\rangle$ is a consequence of the soft mass terms for the $V_{i j}$, induced by SUSY breaking. Since $V_{12}$ and $V_{34}$ have $B-L$ charge +1 and -1 , respectively, we conclude that matter parity is broken dynamically at the low scale $\Lambda$ in the hidden sector.

The only unsuppressed and renormalizable interaction in the superpotential, connecting hidden and visible sector, is given by the term

$$
W \supset-f_{i} \mathcal{Q}_{3}^{\alpha} \mathcal{Q}_{4 \alpha} N_{i}^{c} .
$$

Here, $f_{i}$ with $i=1,2,3$ are free parameters that we will fix below. In the vacuum (2.5) this becomes a linear term for the right-handed neutrino multiplets, which together with the mass term in Eq. (2.2) implies a non-vanishing vacuum expectation value for the corresponding sneutrinos,

$$
\left\langle N_{i}^{c}\right\rangle=\frac{f_{i}}{\lambda_{i}^{S}} \frac{\Lambda^{2}}{M_{S}} .
$$

This vev generates matter parity violating couplings between the standard model fields in the superpotential [28], which are of the order

$$
\begin{array}{rl}
W & \mathcal{O}\left(\frac{\Lambda^{2}}{M_{S}}\right) L H_{u}+\mathcal{O}\left(\frac{\Lambda^{2}}{M_{S} M_{P}}\right) L L E^{c} \\
& +\mathcal{O}\left(\frac{\Lambda^{2}}{M_{S} M_{P}}\right) L Q D^{c}+\mathcal{O}\left(\frac{\Lambda^{2}}{M_{S} M_{P}}\right) D^{c} D^{c} U^{c},
\end{array}
$$

coming from gauge invariant terms like $M_{P}^{-1} N^{c} L L E^{c}$ in the superpotential. Thus the matter-parity breaking is mostly bilinear in our model, originating from the Yukawa couplings in (2.2). Its scale is related to the condensation scale of the hidden sector gauge group. The effects on the matter-parity violation depend on many unknown Yukawa couplings and we will assume a very simple situation where $f_{3} \leq 1$ and $f_{1}=f_{2}=0$ in this paper, to show the presence of a consistent parameter region in the model. Note that we consider that the $N_{1}^{c}$ decay is relevant for leptogenesis. Since $f_{1}=0$, the $N_{1}^{c}$ decays into the hidden sector are suppressed and hence leptogenesis is not affected by the presence of the hidden sector. Furthermore, the matter-parity violating couplings in Eq. (2.8) are small enough to not wash-out the lepton asymmetry again [31] for the parameters we are interested in [1].

When the interactions (2.6) between hidden and visible sector are neglected, we have a global $\mathrm{U}(1)_{B-L}$ symmetry in the hidden sector, since the corresponding gauge-symmetry is already broken by the condensation of $S$ and $\bar{S}$. This global $\mathrm{U}(1)_{B-L}$ symmetry is then broken by $\mathcal{Q}^{\alpha} \mathcal{Q}_{\alpha}$ condensations at the scale $\Lambda$, producing a massless pseudo NG multiplet in the hidden sector. Through the interactions (2.6), this multiplet would receive a supersymmetric mass $m_{\mathrm{NG}}=f_{3}^{2} \frac{\Lambda^{2}}{M_{3}} \sim \mathcal{O}(\mathrm{keV}) \Omega$ In addition, there are hidden sector

\footnotetext{
${ }^{2}$ The true NG boson of the $B-L$ breaking is a linear combination of this pseudo NG boson and the NG boson arises from the high-energy $B-L$ breaking. This true NG boson is nothing but the one absorbed in the $B-L$ gauge field.
} 
multiplets with a mass $\sim \Lambda$. However, after supersymmetry breaking, the soft mass terms raise the masses of the modes in the pseudo NG multiplet. For the pseudo NG boson this yields a mass ( $c f$. app. B]

$$
m_{a} \sim f_{3} \sqrt{m_{3 / 2} \frac{\Lambda^{2}}{M_{3}}} \simeq 100 \mathrm{MeV}_{3}\left(\frac{m_{3 / 2}}{100 \mathrm{GeV}}\right)^{\frac{1}{2}}\left(\frac{\Lambda}{10^{6} \mathrm{GeV}}\right)\left(\frac{M_{3}}{10^{16} \mathrm{GeV}}\right)^{-\frac{1}{2}}
$$

whereas the tree-level masses of the fermion partner $\psi$ and the radial scalar pseudo NG component $\rho$ are given by

$$
m_{\psi} \approx m_{3 / 2}+\mathcal{O}\left(\frac{m_{3 / 2}^{3}}{\Lambda^{2}}\right), \quad m_{\rho} \approx 4 m_{3 / 2}
$$

where we assumed that SUSY breaking is mediated to the hidden sector only via gravity mediation. Below we will always assume that $m_{\psi}>m_{3 / 2}$, since we are interested in gravitino dark matter.

\section{Phenomenology}

In this section we will discuss the decay of the gravitino and the NLSP, which are general features induced by the matter-parity violation, as well as the dynamics of the pseudo NG modes of $B-L$ breaking in the hidden sector, which are special to the model we proposed. The resulting bounds on the parameter space are summarized in Fig. 1 for two reference scenarios.

\subsection{Gravitino and NLSP decay}

As stated above, the matter-parity violating effects on the MSSM sector are mainly induced by the vev of $N_{i}^{c}$ and hence dominated by the bilinear term

$$
W \supset \tilde{\mu}_{i} L_{i} H_{u}
$$

which is generated in the superpotential after condensation of the hidden $\mathrm{SU}(2)_{\text {hid }}$ gauge group as described in the previous section. Here, following Eqs. (2.2) and (2.7), the size of the matter-parity violation is given by

$$
\tilde{\mu}_{i} \simeq f_{3} h_{i 3}^{(n)} \frac{\Lambda^{2}}{M_{3}}=10^{-6} \mathrm{GeV}_{3}\left(\frac{h_{i 3}^{(n)}}{10^{-2}}\right)\left(\frac{\Lambda}{10^{6} \mathrm{GeV}}\right)^{2}\left(\frac{M_{3}}{10^{16} \mathrm{GeV}}\right)^{-1}
$$

where we assumed that $f_{3} \leq 1$ and $f_{1}=f_{2}=0$, as mentioned above.

For the phenomenological analysis, it is convenient to perform a rotation between the higgs- and lepton-doublet superfields:

$$
H_{d} \rightarrow H_{d}-\varepsilon_{i} L_{i}, \quad L_{i} \rightarrow L_{i}+\varepsilon_{i} H_{d},
$$


where $\varepsilon_{i} \equiv \tilde{\mu}_{i} / \mu$, and $\mu$ denotes the MSSM $\mu$-term. The rotation eliminates the bilinear term in Eq. (3.1), but it generates the matter-parity violating trilinear terms $\frac{1}{2} \lambda_{i j k} L_{i} L_{j} E_{k}^{c}$ and $\lambda_{i j k}^{\prime} L_{i} Q_{j} D_{k}^{c}$ with coefficients given by $\lambda_{i j k}=-\varepsilon_{[i,} h_{j] k}^{(e)}$ and $\lambda_{i j k}^{\prime}=-\varepsilon_{i} h_{j k}^{(d)}$, respectively. In general, bilinear $R$-parity breaking terms such as (3.1) imply a small but non-vanishing vev for the left-handed sneutrinos $\tilde{\nu}_{i}$. After the rotation (3.3) it reads $\left\langle\tilde{\nu}_{i}\right\rangle \simeq-\varepsilon_{i} v_{d}\left(1+\mu^{2} / \widetilde{m}_{L i}^{2}\right)$, where $\widetilde{m}_{L i}^{2}$ denotes the soft slepton masses and soft $R$ parity breaking terms were ignored 3 . Note that the operator $\frac{1}{2} \lambda_{i j k}^{\prime \prime} U_{i}^{c} D_{j}^{c} D_{k}^{c}$, which finally leads to proton decay, is not generated in this way. Nevertheless, due to the Plancksuppressed operator $M_{P}^{-1} N^{c} U^{c} D^{c} D^{c}$, it is generically present in the superpotential with coefficients $\lambda^{\prime \prime} \sim \mathcal{O}\left(\Lambda^{2} / M_{P} M_{3}\right)$, cf. Eq. (2.8). Constraints from proton-stability require $\lambda_{11 k}^{\prime} \lambda_{11 k}^{\prime \prime} \lesssim 10^{-27}$ [32], and a sufficient condition to satisfy them is $\Lambda \lesssim 10^{6} \mathrm{GeV}$, where we assumed that $M_{3} \sim 10^{16} \mathrm{GeV}$ and $f_{3}=1$. This tight constrained will be in general relaxed depending on the flavor structure of $\lambda_{i j k}^{\prime \prime}$ and $h_{i j}^{(d)}$ and on the value of $f_{3}$, and we will assume this to be the case below 4

Most importantly, the above violation of matter parity induces the decay of the gravitino [1]. The corresponding decay widths for the two-body decay into $\gamma \nu$ is given by [33]

$$
\Gamma\left(\psi_{3 / 2} \rightarrow \gamma \nu\right)=\frac{1}{32 \pi}\left|U_{\tilde{\gamma} \nu}\right|^{2} \frac{m_{3 / 2}^{3}}{M_{P}^{2}}
$$

where $U_{\tilde{\gamma} \nu}$ parameterizes the photino-neutrino mixing, and $M_{P}=2.4 \times 10^{18} \mathrm{GeV}$ denotes the Planck-mass. Assuming that SUSY breaking only induces matter-parity conserving soft-masses at tree-leve 5 , it can be approximated as [2]

$$
\left|U_{\tilde{\gamma} \nu}\right| \simeq \zeta v g \frac{s_{W}}{\sqrt{2}} \frac{M_{\tilde{W}}-M_{\tilde{B}}}{M_{\tilde{W}} M_{\tilde{B}}} \simeq 10^{-1} \zeta\left(\frac{M_{\tilde{B}}}{150 \mathrm{GeV}}\right)^{-1}
$$

where $\zeta=\frac{v_{d}}{v} \sqrt{\sum \varepsilon_{i}^{2}}$ and $v_{d}=v \cos \beta=\left\langle H_{d}^{0}\right\rangle$ denotes the higgs vev. We made use of the GUT relation $M_{\tilde{W}} / M_{\tilde{B}} \simeq 2$, where $M_{\tilde{B}}$ and $M_{\tilde{W}}$ are the bino and wino masses, respectively. From this, we find for the inverse decay width

$$
\Gamma\left(\psi_{3 / 2} \rightarrow \gamma \nu\right)^{-1} \simeq 4 \times 10^{28} \mathrm{~s}\left(\frac{\tilde{\varepsilon}}{10^{-8}}\right)^{-2}\left(\frac{M_{\tilde{B}}}{150 \mathrm{GeV}}\right)^{2}\left(\frac{\tan \beta}{10}\right)^{2}\left(\frac{m_{3 / 2}}{100 \mathrm{GeV}}\right)^{-3}
$$

\footnotetext{
${ }^{3}$ Vanishing soft bilinear $R$-parity breaking terms can in fact only be demanded at one particular scale. In the generic case without fine tuning bilinear $R$-parity breaking has 9 parameters, whose role for gravitino and NLSP decays was analyzed in detail in 2. Here we ignore phenomenological complications due to the presence of soft bilinears, since in this paper we are mainly interested in the theoretical origin of the supersymmetric term (3.1).

${ }^{4}$ Note that there is also the Planck-suppressed operator $Q Q Q L$ in the superpotential, which leads to proton decay and which is not forbidden by the $B-L$ symmetry. We will assume that this operator is suppressed by some unspecified mechanism, and note that it is not significantly regenerated by breaking of matter-parity.

${ }^{5}$ Note that the above rotation (3.3) regenerates $B h_{u} h_{d}, \widetilde{m}_{L i}^{2} l_{i}^{*} l_{i}$ and $\widetilde{m}_{d}^{2} h_{d}^{*} h_{d}$, which then determine the photino-neutrino mixing angle [2].
} 
with $\tilde{\varepsilon} \equiv \sqrt{\sum \varepsilon_{i}^{2}} \simeq \varepsilon_{3}$, as long as $\varepsilon_{1}, \varepsilon_{2} \lesssim \varepsilon_{3}$. Other decay channels like $\psi_{3 / 2} \rightarrow W^{ \pm} \ell^{\mp}$ and $\psi_{3 / 2} \rightarrow Z^{0} \nu$, and decay channels with the hidden sector pseudo NG boson in the final state, also contribute to the overall decay widths. However, since the branching ratios are of comparable size [1] for the different two-body channels into SM particles, we will concentrate on the decay into gamma-ray lines. This decay channel is constrained by gamma-ray observations, which will give strong bounds on the matter-parity violating parameter $\varepsilon_{3}$ in the region of interest.

The decay of the NLSP, which we take to be the lightest stau $\widetilde{\tau}_{1}$ for definiteness in the rest of the paper, is mediated by the term $L_{i} L_{j} E_{k}^{c}$. For simplicity we will assume that $\widetilde{\tau}_{1}$ is dominantly right-handed, otherwise $L_{i} Q_{j} D_{k}^{c}$ would induce also decays into hadronic jets, see Ref. [1]. The corresponding decay width is dominated by decay into $\nu_{\mu} \tau$ and $\nu_{\tau} \mu$ and given by

$$
\Gamma\left(\widetilde{\tau}_{1} \rightarrow \nu_{\mu} \tau, \nu_{\tau} \mu\right) \simeq 2 \times \frac{1}{16 \pi}\left|\lambda_{233}\right|^{2} m_{\widetilde{\tau}_{1}}
$$

where we neglected fermion masses, and $\lambda_{233}=\varepsilon_{3} h_{23}^{(e)}-\varepsilon_{2} h_{33}^{(e)} \sim-0.1(\tan \beta / 10) \varepsilon_{2}$. From this we obtain for the corresponding decay time

$$
\Gamma\left(\tilde{\tau}_{1} \rightarrow \nu_{\mu} \tau, \nu_{\tau} \mu\right)^{-1} \sim 1 \times 10^{-7} \mathrm{~s}\left(\frac{\varepsilon_{2}}{10^{-8}}\right)^{-2}\left(\frac{10}{\tan \beta}\right)^{2}\left(\frac{m_{\widetilde{\tau}_{1}}}{200 \mathrm{GeV}}\right)^{-1}
$$

from which it follows that the decay of the NLSP can easily happen before BBN. Below we will always assume that $\varepsilon_{2} \simeq \varepsilon_{3} \simeq \varepsilon$ for simplicity.

As mentioned above, a number of astrophysical observations constrain the lifetime of the stau and the gravitino, which translates into bounds on the matter-parity breaking parameters. Firstly, the $\widetilde{\tau}_{1}$ has to decay before BBN, with a lifetime shorter than $2 \times$ $10^{3} \mathrm{~s}$, to avoid catalytic overproduction of ${ }^{6} \mathrm{Li}[13,14]$. This implies the lower limit of roughly $\varepsilon \gtrsim 10^{-13}$. Furthermore, the gravitino decay into gamma-ray lines is limited by observations of the Fermi LAT satellite to lifetimes $\Gamma_{\psi_{3 / 2} \rightarrow \gamma \nu}^{-1} \gtrsim 10^{29} \mathrm{~s}$, yielding an upper limit of $\tilde{\varepsilon} \lesssim 6 \times 10^{-9}\left(m_{3 / 2} / 100 \mathrm{GeV}\right)^{-3 / 2}$ in the mass range where the bounds were evaluated, $60 \mathrm{GeV}<m_{3 / 2}<400 \mathrm{GeV}$ [34]. The corresponding limits on the condensation scale $\Lambda$ are summarized in Fig. 1 for two reference scenario of parameters. In these plots we also show the strong lower bounds on the scale $\Lambda$ and upper bounds on $f_{3}$ that arise from the late decay of the pseudo NG modes of the hidden sector, which we will discuss next.

\subsection{Thermal production of hidden sector particles}

We will now discuss the cosmology of the hidden sector containing the condensing SU(2) hid gauge group. Even when conservatively assuming that reheating only affects the MSSM sector, hidden sector particles are produced in the early universe by scattering of MSSM particles. This can potentially lead to overclosure and can reintroduce problems with BBN. 

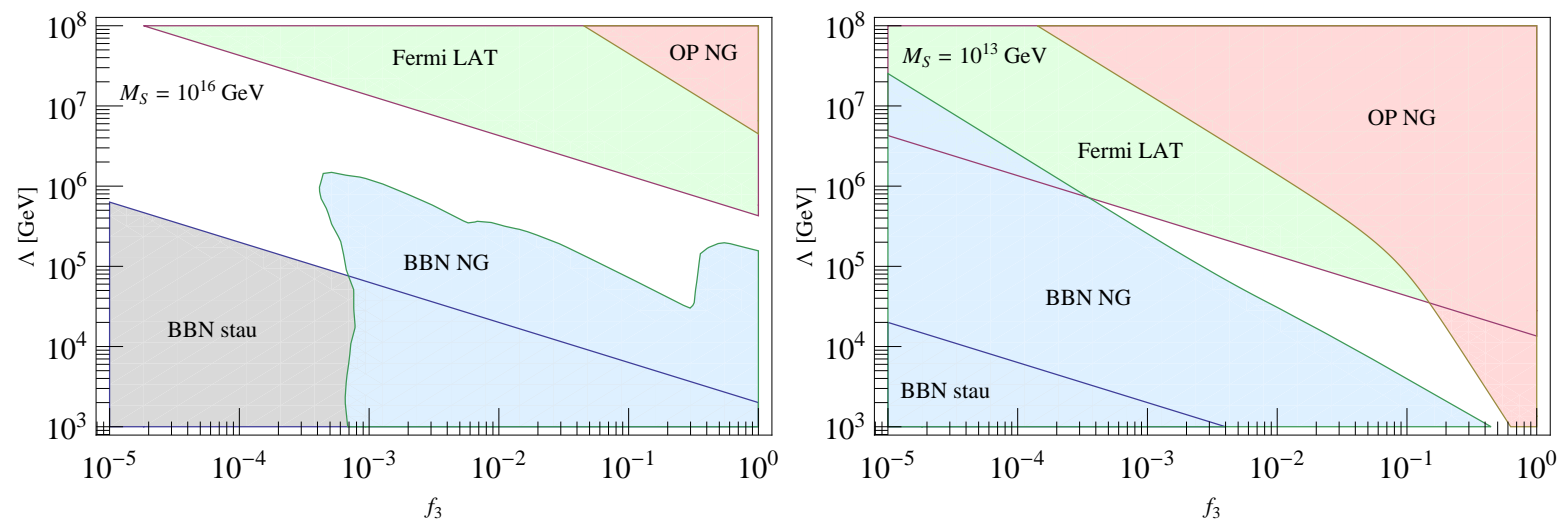

Figure 1: Summary of constraints on condensation scale $\Lambda$, as function of the free parameter $f_{3}$, for two reference scenarios with $M_{S}=10^{16} \mathrm{GeV}$ (left panel) and $M_{S}=10^{13} \mathrm{GeV}$ (right panel). We assumed $m_{3 / 2}=120 \mathrm{GeV}, y=10^{-1}$ and a reheating temperature of $T_{R}=$ $10^{9.8} \mathrm{GeV}$, which yields the correct relic density for the thermally produced gravitino [10]. We show the bounds coming from catalytic production of ${ }^{6} \mathrm{Li}$ during $B B N$ by late decaying staus (BBN stau [14], for stau masses around 150-200 GeV), from the late decay of the fermionic partner of the pseudo NG boson of matter-parity breaking (BBN NG, from Ref. [12], assuming branching ratio into hadrons $\simeq 1$ ), and from the non-observation of gamma-ray lines from gravitino decay by Fermi LAT. For comparison, we show also the region where the pseudo $N G$ boson relic density exceeds the observational limit $\Omega h^{2} \simeq 0.11$ (OP NG). As apparent from the plots, bounds related to the pseudo NG modes become more sever for smaller values of $M_{S}$. We always assumed $f_{1}=f_{2}=0$.

From (2.4) it follows that the hidden sector contains 10 chiral multiplets with a mass at the condensation scale, $\sim \Lambda$, as well as one chiral multiplet that remains light and is the pseudo NG mode of the breaking of the accidental global $B-L$ symmetry in the hidden sector. As mentioned above, the pseudo NG boson $a$ acquires a small mass term, suppressed by $M_{S}$, due to the coupling to the $B-L$ breaking visible sector, see (2.9), whereas the fermionic partner $\psi$ and bosonic partner $\rho$ of the pseudo NG boson get masses around the gravitino mass $m_{3 / 2}$, see (2.10).

At temperatures above the condensation scale, $T \gg \Lambda$, the production of hidden sector particles is dominated by the $M_{S}$-suppressed dim-5 operator

$$
W \supset \frac{y}{M_{3}} f_{3} \mathcal{Q}_{3} \mathcal{Q}_{4} L H_{u},
$$

which is generated after integrating out $N_{3}^{c}$, and by the $M_{S}^{2}$ suppressed dim- 6 operators coming from integrating out the heavy $B-L$ vector-multiplet, which gives operators like

$$
K \supset \frac{\left(\mathcal{Q}_{1}^{\dagger} \mathcal{Q}_{1}+\mathcal{Q}_{2}^{\dagger} \mathcal{Q}_{2}\right) L^{\dagger} L}{M_{S}^{2}}
$$

in the Kahler potential $K$ [35]. Here we have defined $y=\max \left(h_{13}^{(n)}, h_{23}^{(n)}, h_{33}^{(n)}\right)$, and we will ignore the flavor structure for simplicity. Note that the term (3.9) violates the $\mathrm{U}(1)_{\mathrm{B}-\mathrm{L}}$ 
symmetry explicitly, but since this interaction between hidden and visible sector is never in equilibrium, the lepton asymmetry produced by leptogenesis is maintained.

In the first case, the matrix element for $2 \rightarrow 2$ scattering processes like $l h_{u} \rightarrow \mathcal{Q}_{3} \mathcal{Q}_{4}$ is of the order $|\mathcal{M}|^{2} \sim y^{2} f_{3}^{2} s / M_{3}^{2}$, where $s$ denotes the center-of-mass energy. Following Ref. [36], the yield $Y_{\mathcal{Q}_{i}}=n_{\mathcal{Q}_{i}} / S$ of hidden sector quarks $\mathcal{Q}_{i}$, where $S$ is the entropy density, is given by

$$
Y_{\mathcal{Q}_{i}}^{\mathrm{UV}, W} \sim y^{2} f_{3}^{2} \frac{T_{R} M_{P}}{\pi^{7} M_{3}^{2} g_{*}^{3 / 2}} \simeq 2 \times 10^{-12} y^{2} f_{3}^{2}\left(\frac{T_{R}}{10^{9} \mathrm{GeV}}\right)\left(\frac{M_{3}}{10^{16} \mathrm{GeV}}\right)^{-2}
$$

where we have used $g_{*} \simeq 915 / 4$ as the number of effective relativistic degrees of freedom of the MSSM. Similarly, in the second case, the operator in (3.10) induces $2 \rightarrow 2$ scattering processes like $l^{*} l \rightarrow \mathcal{Q}_{1}^{*} \mathcal{Q}_{1}$, and the corresponding matrix element is of the order $|\mathcal{M}|^{2} \sim$ $s^{2} / M_{S}^{4}$. The corresponding yield is given by

$$
Y_{\mathcal{Q}_{i}}^{\mathrm{UV}, K} \sim \frac{T_{R}^{3} M_{P}}{\pi^{7} M_{S}^{4} g_{*}^{3 / 2}} \sim 10^{-26}\left(\frac{T_{R}}{10^{9} \mathrm{GeV}}\right)^{3}\left(\frac{M_{S}}{10^{16} \mathrm{GeV}}\right)^{-4} .
$$

The above processes are most efficient at the highest temperature, $T_{R}$. The energy density produced in the hidden sector can be hence estimated to be $\rho_{\mathrm{HS}} \sim T_{R} n_{\mathrm{HS}}$, where $n_{\mathrm{HS}}=Y_{\mathcal{Q}_{i}}^{\mathrm{UV}} S$ denotes the particle density in the hidden sector shortly after reheating. Assuming that particles in the hidden sector quickly thermalize, this corresponds to a temperature $T_{\mathrm{HS}}$ in the hidden sector that is given by $\left(\pi^{2} / 30\right) g_{\mathrm{HS}}^{(R)} T_{\mathrm{HS}}^{4}=\rho_{\mathrm{HS}}$ [37]. From this it follows that the temperature in the hidden sector shortly after reheating is $T_{\mathrm{HS}} \sim$ $\left(g_{\mathrm{VS}}^{(R)} / g_{\mathrm{HS}}^{(R)}\right)^{1 / 4}\left(Y_{\mathcal{Q}_{i}}^{\mathrm{UV}}\right)^{1 / 4} T_{R}$, where $g_{\mathrm{HS}}^{(R)}$ and $g_{\mathrm{VS}}^{(R)}$ denote the corresponding effective degrees of freedom in the hidden and visible sector at high energies.

Depending on the details of the symmetries of the hidden sector, some of the heavy hidden sector states with masses around the condensation scale $\sim \Lambda$ can be stable, which could lead to overclosure of the universe. However, despite their large masses, which can exceed the unitarity bound $\Lambda \lesssim 10^{5} \mathrm{GeV}$ [38], sufficiently strong annihilation of the hidden sector states is in principle possible due to the large temperature difference between hidden and visible sector, which reduces the relic density by a factor of order $T_{\mathrm{HS}} / T_{\mathrm{VS}} \sim\left(Y_{\mathcal{Q}_{i}}^{\mathrm{UV}}\right)^{\frac{1}{4}}$, see Ref. [39]. However, a detailed study of this annihilation process is beyond the scope of this paper. Subsequently we will assume for simplicity that all these potentially stable heavy hidden sector states efficiently annihilate into the pseudo NG modes, and we will consider the subsequent effects of these NG modes only. Alternatively, depending on the underlying symmetries, the heavy hidden sector particles could directly decay into Standard Model particles before BBN, thus avoiding the overclosure problems from the beginning. We will discuss this situation shortly in section 4 below.

To estimate the hidden sector temperature and density of pseudo NG modes at later times, one can make use of the fact that the entropy densities, which is given by $S_{\mathrm{HS} / \mathrm{VS}} \simeq\left(2 \pi^{2} / 45\right) g_{\mathrm{HS} / \mathrm{VS}} T_{\mathrm{HS} / \mathrm{VS}}^{3}$, in the hidden and visible sector are conserved separately [37]. $>$ From this it follows that today $T_{\mathrm{HS}} \sim\left(g_{\mathrm{VS}} / g_{\mathrm{HS}}\right)^{1 / 3}\left(Y_{\mathcal{Q}_{i}}^{\mathrm{UV}}\right)^{1 / 4} T_{\mathrm{VS}}$, where $g_{\mathrm{VS}}$ and 
$g_{\mathrm{HS}}$ denote the corresponding effective degrees of freedom of the hidden and visible sector, now at low energies. Noting that the particle density today is given by $n_{\mathrm{HS}} \approx g_{\mathrm{HS}} / \pi^{2} T_{\mathrm{HS}}^{3}$, this gives an effective particle yield of $Y_{\mathrm{NG}}^{\mathrm{UV}}=n_{\mathrm{HS}} / S_{\mathrm{VS}} \sim 0.2\left(Y_{\mathcal{Q}_{i}}^{\mathrm{UV}}\right)^{3 / 4}$. The corresponding relic density of the different pseudo NG modes is given by

$$
\Omega_{a, \rho, \psi}^{\mathrm{UV}} h^{2}=2.74 \times 10^{10}\left(\frac{m_{a, \rho, \psi}}{100 \mathrm{GeV}}\right) Y_{a, \rho, \psi}^{\mathrm{UV}},
$$

and we take for definiteness $4 Y_{a}^{\mathrm{UV}}=4 Y_{\rho}^{\mathrm{UV}}=2 Y_{\psi}^{\mathrm{UV}}=Y_{\mathrm{NG}}^{\mathrm{UV}}$.

At temperatures below the condensation scale, $100 \mathrm{GeV} \lesssim T \ll \Lambda$, hidden sector particles are predominantly produced via the renormalizable operator

$$
W \supset y f_{3} \frac{\Lambda}{M_{3}} V_{34} L H_{u} .
$$

Here, the decay of particles in the thermal bath into the hidden sector particles is relevant, like e.g. decays of the higgs $h^{0} \rightarrow \psi \nu$. The corresponding relic density is given by [36]

$$
\Omega_{a, \rho, \psi}^{I R} h^{2} \approx \frac{10^{27}}{g_{*}^{3 / 2}} m_{a, \rho, \psi} \sum_{j} \frac{\Gamma_{j}}{m_{j}^{2}} \sim 10^{2} f_{3}^{2} y^{2}\left(\frac{m_{a, \rho, \psi}}{100 \mathrm{GeV}}\right)\left(\frac{\Lambda}{10^{6} \mathrm{GeV}}\right)^{2}\left(\frac{M_{3}}{10^{16} \mathrm{GeV}}\right)^{-2}
$$

where $\Gamma_{j} \sim 1 / 8 \pi\left(y f_{3} \Lambda / M_{3}\right)^{2} m_{j}$ denotes the approximate decay widths of particles $j$ in the bath into the hidden sector modes, and we used $g_{*} \approx 100$. This can dominate over (3.13) when $\Lambda$ is large.

\subsection{Decay of the hidden sector pseudo NG modes}

Finally, we discuss the decay of the pseudo NG modes. The fermionic pseudo NG boson partner $\psi$ mixes with the neutrinos $\nu_{i}$ and inherits their SM interactions. This mixing is induced by the term (2.6), and given by (for details see app. A)

$$
\theta_{i} \simeq \frac{h_{i 3}^{(n)} f_{3}}{\sqrt{2}} \frac{v_{u} \Lambda}{M_{3} m_{\psi}}
$$

which is suppressed by the $B-L$ breaking scale $M_{S}$. Here, the index $i$ refers to neutrino flavor eigenstates. Using (3.2), the size of the mixing angle can be expressed in term of the matter-parity breaking parameter like

$$
\theta_{i} \simeq 1.2 \times 10^{-13}\left(\frac{\tilde{\mu}_{i}}{10^{-7} \mathrm{GeV}}\right)\left(\frac{m_{\psi}}{100 \mathrm{GeV}}\right)^{-1}\left(\frac{\Lambda}{10^{6} \mathrm{GeV}}\right)^{-1} .
$$

If $m_{\psi}>M_{Z}$, the two-body decay $\psi \rightarrow Z^{0} \nu_{i}$ is kinematically allowed, and the corresponding decay width is given by

$$
\Gamma\left(\psi \rightarrow Z^{0} \nu_{i}\right) \simeq \frac{e^{2}}{16 \pi} \frac{\theta_{i}^{2}}{8 s_{W}^{2} c_{W}^{2}} \frac{m_{\psi}^{3}}{M_{Z}^{2}}\left(1+\frac{M_{Z}^{2}}{m_{\psi}^{2}}-2 \frac{M_{Z}^{4}}{m_{\psi}^{4}}\right)\left(1-\frac{M_{Z}^{2}}{m_{\psi}^{2}}\right)
$$




$$
\simeq(0.03 \mathrm{~s})^{-1}\left(\frac{\theta_{i}}{10^{-11}}\right)^{2}\left(\frac{m_{\psi}}{150 \mathrm{GeV}}\right) \frac{m_{\psi}^{2}}{M_{Z}^{2}}\left(1+\frac{M_{Z}^{2}}{m_{\psi}^{2}}-2 \frac{M_{Z}^{4}}{m_{\psi}^{4}}\right)\left(1-\frac{M_{Z}^{2}}{m_{\psi}^{2}}\right)
$$

For masses around $m_{\psi} \sim 150 \mathrm{GeV}$ and mixing angles $\theta_{i} \sim 10^{-11}$ this gives inverse decay widths of the order of $\tau \sim 0.03 \mathrm{~s}$, which is well before and save for BBN [12]. However, when $\theta_{i}$ is smaller, BBN bounds become very relevant 6 The lifetime of $\psi$ is further reduced by the decay channels $\psi \rightarrow W^{ \pm} \ell^{\mp}$, whose decay width is obtained from (3.18) by $\Gamma_{\psi \rightarrow W^{ \pm} \ell_{i}^{\mp}}=4 c_{W}^{2} \Gamma_{\psi \rightarrow Z^{0} \nu_{i}}$ and substituting $M_{Z} \rightarrow M_{W^{ \pm}}$. Note, that if $m_{\psi}<M_{W^{ \pm}}$, the much slower three-body decays like $\psi \rightarrow e^{+} e^{-} \nu$ would become important. They would give rise to much stronger lower bounds on the mixing angle $\theta$ than in the regime where two-body decay is allowed.

The bosonic pseudo NG boson partner $\rho$ can perform the two-body decays like $\rho \rightarrow \tilde{h}_{u} \nu$, if kinematically allowed, as follows from the effective operator (3.9). This requires that $M_{\tilde{h}_{u}}<m_{\rho}$. The lifetime of $\rho$ is typically much smaller than the lifetime of $\psi$, since its decay is not further suppressed by gauge couplings. When the above decay is kinematically forbidden, three-body decays like $\rho \rightarrow b \bar{b} \nu$ become relevant. However, this depends on the details of the MSSM spectrum, and we will conservatively assume that the decay of $\rho$ is always faster than the decay of $\psi$.

The pseudo NG boson $a$ in principle also decays into SM particles. The interactions with the visible sector are at low energies mainly mediated by the operators $V_{34} L_{i} H_{u}$, and therefore the interaction is suppressed only by a factor $h_{i 3}^{(n)} \Lambda / M_{3}$. However, since the particle interacts with the visible sector only via a matter parity violating operator, final states for its decay can only be odd under matter parity. But no matter parity odd final states with integer spin number exists below the mass of the lightest gaugino, and hence the particle is essentially stable and one has to require that $\Omega_{a}^{\mathrm{IR}, \mathrm{UV}} h^{2} \lesssim 0.1$ to avoid overclosure 7

Lastly, we note that the invisible decay of gravitinos into the pseudo NG boson and neutrinos, which is kinematically allowed, happens with lifetimes around $\tau \sim 10^{32} \mathrm{~s}\left(10^{-12} / \theta\right)^{2}$ for $\sim 100 \mathrm{GeV}$ masses, and can hence be neglected. Furthermore, the decay $\tilde{\tau} \rightarrow \tau \psi$, which is also in principle possible, is double suppressed by the small matter-parity breaking $\tilde{\mu} / \mu$, and hence negligible with respect to the decay mode $\tilde{\tau} \rightarrow \tau \nu$.

The bounds on the parameter space of our model are summarized in Fig. 1 for two reference scenarios. We show bounds from the non-observation of gamma-ray lines with Fermi LAT, where we have assumed that gravitinos make essentially all of dark matter today. Furthermore, we show bounds from BBN that stem from the late decay of the stau via the induced $\mathrm{R}$-parity violation, and bounds from BBN that come from the late decay

\footnotetext{
${ }^{6}$ Note that the decay into gravitinos, $\psi \rightarrow a \psi_{3 / 2}$, is typically very slow with lifetimes of the order of $\tau \gtrsim 10^{8} \mathrm{~s}$ or larger, and can be safely neglected.

${ }^{7}$ In fact, the decay rate is doubly suppressed by the small matter-parity violation, and loop suppressed since the decay involves the higgs-multiplets, e.g. $\Gamma(a \rightarrow \gamma \gamma) \sim \alpha^{2}\left(y f_{3} \Lambda / M_{3} \tilde{\mu} / \mu\right)^{2} m_{a}^{5} / \mu^{4} \sim \mathcal{O}\left(10^{42} \mathrm{~s}\right)^{-1}$.
} 
of the fermionic and bosonic pseudo NG boson partner. Lastly, we also show the region where the relic density of the pseudo NG boson would exceed the observational limit.

\section{Discussion}

As shown in Fig. 1, cosmologically viable models with high reheating temperatures and gravitino dark matter can be found for large enough $\mathrm{U}(1)_{B-L}$ breaking scales $M_{S} \gtrsim$ $\mathcal{O}\left(10^{13} \mathrm{GeV}\right)$ and $\mathrm{SU}(2)_{\text {hid }}$ condensation scales $\Lambda \sim \mathcal{O}\left(10^{3}-10^{8} \mathrm{GeV}\right)$. We also assumed suppressed couplings between hidden sector and the lighter two right-handed neutrinos, adopting $f_{1}=f_{2}=0$ for simplicity. In these models, the communication between the matter-parity breaking $\mathrm{SU}(2)_{\text {hid }}$ sector and the visible sector is suppressed by the mass of the heaviest right-handed neutrino, $M_{3} \simeq M_{S}$. As an important consequence, the mass of the pseudo NG boson of the breaking of the accidental global $B-L$ symmetry in the hidden sector, as well as its relic number density, are small enough to yield a relic density in agreement with the observations, so that the pseudo NG boson constitutes only a subdominant component of dark matter. Furthermore, the relic density and the lifetime of the pseudo NG boson partners can be small enough to be in agreement with the tight BBN constraints. Note that requiring that the lighter two right-handed neutrinos do not change the above phenomenology implies upper limits on $f_{1}$ and $f_{2}$ that are at least of the order $f_{i} \lesssim f_{3} M_{i} / M_{3}$, since the interactions between hidden and visible sector depends on the ratio $f_{i} / M_{i}$.

Due to the strong BBN bounds on the relic abundance and the lifetime of the pseudo NG modes of $B-L$ breaking, large values of matter-parity breaking are favored in our model. This implies both a lifetime of the gravitino closer to the experimental limit, as well as shorter decay lengths of the NLSPs. For values of matter-parity breaking close to the Fermi LAT limit, we estimate the decay length of the stau to be of the order of $\mathcal{O}(100 \mathrm{~m})$. It is very interesting, that this is in principle accessible by the LHC [40]. However, the quantitative details depend e.g. on the flavor structure of the stau couplings, and we refer to Ref. [2] for a detailed discussion.

In general, the low-scale breaking of discrete symmetries can induce serious cosmological domain wall problems, which arise after the completion of inflation and thus are never diluted [41]. In the presented scenario these problems can be avoided in two different ways.

Firstly, estimating the maximum temperature $T_{\mathrm{HS}}$ of the hidden sector immediately after reheating, by equating $\rho_{\mathrm{HS}} \sim T_{\mathrm{VS}} n_{\mathrm{VS}} Y \sim T_{\mathrm{HS}}^{4}$, shows that the hidden sector temperature after inflation does never exceed the breaking scale $\Lambda$ in a large part of the allowed parameter space in Fig. 1 (namely for $f_{3} \lesssim 0.1$ in case of $M_{S}=10^{16} \mathrm{GeV}$ ). In these cases, the matter-parity symmetry is never restored after inflation, despite its low breaking scale, thus avoiding all domain wall problems.

Secondly, even if the symmetry is restored, domain wall problems can be in principle avoided if the discrete symmetry is embedded into an $\mathrm{U}(1)$ gauge symmetry, since in that case the minima of the potential are continuously connected and the domain walls are 


\begin{tabular}{c|cccc|cccc|c} 
& $\mathcal{Q}_{1}^{\alpha}$ & $\mathcal{Q}_{2}^{\alpha}$ & $\mathcal{Q}_{3}^{\alpha}$ & $\mathcal{Q}_{4}^{\alpha}$ & $Z_{13}$ & $Z_{14}$ & $Z_{23}$ & $Z_{24}$ & $X$ \\
\hline \hline $\mathrm{U}(1)_{B-L}$ & $\frac{1}{2}$ & $\frac{1}{2}$ & $-\frac{1}{2}$ & $-\frac{1}{2}$ & 0 & 0 & 0 & 0 & 0 \\
\hline $\mathrm{U}(1)_{a}^{(\text {acc. })}$ & $\frac{1}{2}$ & $-\frac{1}{2}$ & $\frac{1}{2}$ & $-\frac{1}{2}$ & -1 & 0 & 0 & 1 & 0 \\
$\mathrm{U}(1)_{b}^{(\text {acc. })}$ & $\frac{1}{2}$ & $-\frac{1}{2}$ & $-\frac{1}{2}$ & $\frac{1}{2}$ & 0 & -1 & 1 & 0 & 0 \\
$\mathrm{U}(1)_{R}^{(\text {acc. })}$ & $-\frac{1}{2}$ & $-\frac{1}{2}$ & $\frac{1}{2}$ & $\frac{1}{2}$ & 2 & 2 & 2 & 2 & 2
\end{tabular}

Table 4.1: The global symmetries of the superpotential (2.4). The standard model fields are not charged under $U(1)_{a, b}^{(\text {acc. })}$, whereas their charges with respect to $U(1)_{B-L}$ and $U(1)_{R}^{(\text {acc. })}$ are the canonical ones. The fields $S, \bar{S}$ are neutral with respect to the accidental symmetries.

no longer stable. This mechanism requires however that the gauge symmetry is broken after inflation, since only then the domain walls are bounded by cosmic strings and they shrink and decay rapidly when the $\mathrm{U}(1)$ is spontaneously broken after inflation ends [42]. Therefore, in cases where the $\mathrm{U}(1)_{B-L}$ breaking occurs after inflation, our model is free of cosmological domain wall issues, despite the low scale breaking.

The superpotential (2.4) has four global U(1) symmetries, with charge assignments as listed in Table 4.1. Besides $\mathrm{U}(1)_{B-L}$ it exhibits three additional accidental symmetries, $\mathrm{U}(1)_{a}^{(\text {acc. })}, \mathrm{U}(1)_{b}^{(\text {acc. })}$ and the $R$-symmetry $\mathrm{U}(1)_{R}^{(\text {acc. })}$. They reflect the absence of linear terms and quadratic terms for the fields $Z_{i j}$ in the superpotential. These symmetries are either explicitly broken by higher order operators, for example trilinear terms which do not influence the vacuum (2.5), or they are symmetries of the full Lagrangian. In the latter case one may gauge the symmetries, since they are anomaly free. However, we do not want to have additional massless gauge fields in the model, and hence we rather consider unbroken discrete subgroups of these symmetries in that case.

The accidental $R$-symmetry disappears if one introduces mass terms like $W=M Z_{13} Z_{24}$ in the superpotential. Such terms are compatible 8 with the vacuum under consideration. However, if one wants to avoid the prediction of light fermions with masses $\sim \Lambda^{2} / M$, generated by a see-saw like mechanism in the hidden sector, we need that $M \ll \Lambda$. This may follow if the explicit violation of $\mathrm{U}(1)_{R}^{(\text {acc.) }}$ occurs only via higher-dimensional operators in the hidden sector. Alternatively, one may consider that the fields $Z_{i j}$ are moduli of an underlying theory, and therefore have no mass terms. Finally, note that the combination $R^{\text {acc. }}+B-L$ is orthogonal to the vevs $\left\langle V_{12}\right\rangle,\left\langle V_{34}\right\rangle$. After supersymmetry breaking this symmetry is broken to its $\mathbb{Z}_{2}$ subgroup, which is trivial up to hypercharge.

The absence of linear terms $W=C_{i j} Z_{i j}$ is crucial for our model, since such terms completely remove the vacuum (2.5). It can be explained by promoting a discrete subgroup of $\mathrm{U}(1)_{a, b}^{\text {acc. }}$ to a rigorous symmetry of the hidden sector. An important consequence is then that the lowest mass states of $V_{13}, V_{14}, V_{23}$ and $V_{24}$ become stable together with $Z_{i j}$ due to the $\mathrm{U}(1)_{a, b}^{(\text {acc.) }}$ symmetry. As discussed above, this can be problematic since their relic

\footnotetext{
${ }^{8}$ In addition new supersymmetric minima with $\langle X\rangle= \pm \Lambda^{2} / M$ occur, where $B-L$ remains unbroken.
} 
mass density may overclose the universe, if annihilation into the pseudo NG multiplet is not efficient enough. In this cases it would be better to break the $\mathrm{U}(1)_{a, b}$ symmetries explicitly, to allow the decay of the hidden sector particles. As an example, we note that the linear combination $\mathrm{U}(1)_{a+b}$ alone is needed in order to forbid the linear terms in $Z_{i j}$. This combination is preserved if we enlarge (2.4) to

$$
W_{\mathrm{dyn}}=X\left(\operatorname{Pf}\left(V_{i j}\right)-\Lambda^{2}\right)+\Lambda V_{13}\left(Z_{13}+Z_{14}\right)+\ldots+\Lambda V_{24}\left(Z_{23}+Z_{24}\right) .
$$

We can then gauge $\mathrm{U}(1)_{a+b}$ and assume it is spontaneously broken to a $\mathbb{Z}_{2}$ subgroup at a high scale $\Lambda^{\prime}$, such that the heavy hidden sector states have odd parity. If we now identify this $\mathbb{Z}_{2}$ with the Peccei-Quinn like $\mathbb{Z}_{2}$ of the SSM (cf. [26], in SU(5) notation)9]

$$
P(\mathbf{1 0})=P\left(H_{d}\right)=-, \quad P\left(5^{*}\right)=P\left(H_{u}\right)=P\left(N^{c}\right)=+,
$$

decay channels of the hidden sector states to $H_{u} H_{d}$ open up. This can resolve the overclosing issue of the hidden sector from the start. Since the breaking of this $\mathbb{Z}_{2}$ is then related to the generation of the $\mu$ term, we expect that also the linear terms in $Z_{i j}$ remain small, since they are additionally suppressed by the scale $\Lambda^{\prime}$.

\section{Conclusions}

In this paper we have discussed a possible mechanism that generates a small matter-parity violation in the visible sector. Such a violation of matter-parity can resolve the tension between gravitino LSPs and high reheating temperatures as required by thermal leptogenesis. In our scenario, visible sector and part of the hidden sector are simultaneously charged under a gauged $\mathrm{U}(1)_{B-L}$. This $\mathrm{U}(1)_{B-L}$ is broken at a high scale $M_{S}$ by right-handed neutrino masses to its matter-parity $\mathbb{Z}_{2}$ subgroup. Matter-parity is then subsequently broken completely in the hidden sector by a $\mathrm{SU}(2)_{\text {hid }}$ quark condensate at a scale $\Lambda$. This mechanism induces a small vev $\sim \Lambda^{2} / M_{S}$ for the right-handed neutrinos, giving rise to bilinear matter-parity breaking in the visible sector. Due to matter-parity breaking, the NLSP, typically a stau or neutralino, can then decay into standard model particles before conflicting with BBN.

Since the hidden sector is almost separated from the high-energy $B-L$ breaking sector, the hidden sector possesses an accidental global $B-L$ symmetry which is explicitly broken only through the interactions with the right-handed neutrinos. The effects of the explicit breaking are suppressed by $1 / M_{S}$ at low energies, and hence the pseudo NG modes arising from the $B-L$ breaking in the hidden sector acquire only very small masses. The tightest constraints on our proposed model come from the dynamics of these pseudo NG modes. The pseudo NG boson itself acquires $\mathcal{O}(\mathrm{MeV}-\mathrm{GeV})$ masses, remains practically stable, and can be easily overproduced if the coupling between hidden and visible sector is too large. The fermionic and bosonic partners of the pseudo NG boson, however, acquire $\mathcal{O}(100 \mathrm{GeV})$ masses, decay early in the universe and can reintroduce problems with BBN. We showed

\footnotetext{
${ }^{9}$ This $\mathbb{Z}_{2}$ forbids the dangerous superpotential operators $101010 \mathbf{5}^{*}, \mathbf{1 0} \mathbf{5}^{*} \mathbf{5}^{*}$, but not $\mathbf{5}^{*} H_{u}$.
} 
that the model can be phenomenologically viable for large enough $B-L$ breaking scale $M_{S} \gtrsim \mathcal{O}\left(10^{13} \mathrm{GeV}\right)$, and for condensation scales $\Lambda \sim \mathcal{O}\left(10^{3}-10^{8} \mathrm{GeV}\right)$, when the coupling to the lighter right-handed neutrinos is suppressed.

An important consequence of gravitino dark matter scenarios with small breaking of matter-parity is that the gravitino decays, producing gamma-ray lines which are potentially observable with instruments like Fermi LAT. We found that within our model, large values of the matter-parity violation are favored, since this reduces the lifetime of the problematic pseudo NG modes. This suggests lifetimes of the gravitino close to the experimental limit, as well as short decay lengths of the NLSP. For a stau NLSP, we found a lower limit for typical stau decay lengths that is given by $\gtrsim \mathcal{O}(100 \mathrm{~m})$. Such events are in principle detectable at the LHC.

\section{Acknowledgements}

We would like to thank Wilfried Buchmuller and Jan Hajer for useful discussions. JS and CW would like to thank the IPMU for kind hospitality during the main stages of this work. This work was supported by the World Premier International Research Center Initiative (WPI Initiative), MEXT, Japan.

\section{A Fermion Mixing Matrix}

The superpotential contributions (2.2), (2.4) and (2.6) imply mixings between the 13 neutral fermions

$$
\tilde{B}, \tilde{W}, \tilde{h}_{u}^{0}, \tilde{h}_{d}^{0}, \nu_{i}, N_{i}^{c}, \tilde{V}_{12}, \tilde{V}_{34}, \tilde{X},
$$

where the first four fields are the MSSM gauginos and neutral higgsinos, $\nu_{i}$ and $N_{i}^{c}$ are the left- and right-handed neutrinos, respectively, and the last three fields are fermions of hidden sector chiral multiplets. In this basis the mass matrix is to leading order (and up to order one coefficients)

$\hat{M}=\left(\begin{array}{cccc|cc|ccc}M_{B} & 0 & M_{Z} & -M_{Z} & \alpha_{i} \Lambda^{2} / M_{S} & 0 & 0 & 0 & 0 \\ 0 & M_{W} & -M_{Z} & M_{Z} & \alpha_{i} \Lambda^{2} / M_{S} & 0 & 0 & 0 & 0 \\ M_{Z} & -M_{Z} & 0 & -\mu & \beta_{i} \Lambda^{2} / M_{S} & \alpha_{i} \Lambda^{2} / M_{S} & 0 & 0 & 0 \\ -M_{Z} & M_{Z} & -\mu & 0 & 0 & 0 & 0 & 0 & 0 \\ \hline \alpha_{i} \Lambda^{2} / M_{S} & \alpha_{i} \Lambda^{2} / M_{S} & \beta_{i} \Lambda^{2} / M_{S} & 0 & 0 & h_{i j}^{(n)} v_{u} & 0 & 0 & 0 \\ 0 & 0 & \alpha_{i} \Lambda^{2} / M_{S} & 0 & h_{j i}^{(n)} v_{u} & \lambda_{i j}^{S} M_{S} & 0 & \delta_{i 3} \Lambda & 0 \\ \hline 0 & 0 & 0 & 0 & 0 & 0 & 0 & m_{\psi} & \Lambda \\ 0 & 0 & 0 & 0 & 0 & \delta_{i 3} \Lambda & m_{\psi} & 0 & \Lambda \\ 0 & 0 & 0 & 0 & 0 & 0 & \Lambda & \Lambda & 0\end{array}\right)$, 
where $\xi \equiv \Lambda / M_{P}$ and $v_{u}$ denotes the vev of the bosonic component of the superfields $H_{u}$, and we took $f_{3}=1$ and $f_{1}=f_{2}=0$ for definiteness. The coefficients $\alpha_{i}$ and $\beta_{i}$ are non-zero for non-vanishing vevs of the the left- and right-handed sneutrinos, respectively.

The $4 \times 4,6 \times 6$ and $3 \times 3$ blocks on its diagonal correspond to the MSSM neutralinos, the left- and right-handed neutrinos and three hidden sector fermions. In the basis in which the neutralino and the hidden sector mass matrices are diagonal and the left and right-handed neutrinos decouple, the matrix reads (only leading order terms are shown)

$$
\hat{M} \sim\left(\begin{array}{c|cc|ccc}
M_{\chi \alpha \beta} & \tilde{\alpha}_{\alpha i} \Lambda^{2} / M_{S} & \tilde{\beta}_{\alpha i} \Lambda^{2} / M_{S} & 0 & 0 & 0 \\
\hline \tilde{\alpha}_{i \alpha} \Lambda^{2} / M_{S} & M_{\nu_{L} i j} & 0 & -h_{i 3}^{(n)} v_{u} \Lambda / \sqrt{2} M_{3} & h_{i 3}^{(n)} v_{u} \Lambda / 2 M_{3} & h_{i 3}^{(n)} v_{u} \Lambda / 2 M_{3} \\
\tilde{\beta}_{i \alpha} \Lambda^{2} / M_{S} & 0 & M_{\nu_{R} i j} & \delta_{i 3} v_{u} / \sqrt{2} & -\delta_{i 3} v_{u} / 2 & -\delta_{i 3} v_{u} / 2 \\
\hline 0 & -h_{3 i}^{(n)} v_{u} \Lambda / \sqrt{2} M_{3} & \delta_{3 i} v_{u} / \sqrt{2} & -m_{\psi} & 0 & 0 \\
0 & h_{3 i}^{(n)} v_{u} \Lambda / 2 M_{3} & -\delta_{3 i} v_{u} / 2 & 0 & -\sqrt{2} \Lambda & 0 \\
0 & h_{3 i}^{(n)} v_{u} \Lambda / 2 M_{3} & -\delta_{3 i} v_{u} / 2 & 0 & 0 & \sqrt{2} \Lambda
\end{array}\right),
$$

with neutrino mass matrices $M_{\nu_{L} i j}=-v_{u}^{2} \sum_{k} h_{i k}^{(n)} h_{k j}^{(n) T} / M_{k}, M_{\nu_{R} i j}=M_{i} \delta_{i j}+\mathcal{O}\left(v_{u}^{2} / M_{S}\right)$, and diagonal neutralino masses $M_{\chi \alpha \beta}=m_{\chi_{\alpha}} \delta_{\alpha \beta}, \alpha, \beta=1, \ldots, 4$.

We deduce that the left-handed neutrinos mix with lightest neutralino $\chi_{1}$ and the light pseudo NG fermion $\Psi$ as

$$
\begin{aligned}
& \theta_{\nu_{L} \chi_{1}} \sim \frac{\Lambda^{2}}{M_{S} m_{\chi_{1}}} \sim 5 \times 10^{-7}\left(\frac{\Lambda}{10^{6} \mathrm{GeV}}\right)^{2}\left(\frac{M_{S}}{10^{16} \mathrm{GeV}}\right)^{-1}\left(\frac{m_{\chi_{1}}}{200 \mathrm{GeV}}\right)^{-1} \\
& \theta_{\nu_{L i} \Psi} \simeq \frac{h_{i 3}^{(n)}}{\sqrt{2}} \frac{\Lambda v_{u}}{M_{S} m_{\psi}} \simeq 1.2 \times 10^{-12}\left(\frac{h_{i 3}^{(n)}}{10^{-2}}\right)\left(\frac{\Lambda}{10^{6} \mathrm{GeV}}\right)\left(\frac{M_{S}}{10^{16} \mathrm{GeV}}\right)^{-1}\left(\frac{m_{\psi}}{100 \mathrm{GeV}}\right)^{-1} .
\end{aligned}
$$

\section{B Pseudo NG mode masses}

We will shortly discuss how the pseudo NG modes of $B-L$ breaking in the hidden sector acquire masses after SUSY breaking. To this end we will assume that SUSY breaking is only mediated by SUGRA effects to the matter-parity breaking sector, and that the Kahler potential is canonical.

The mass-generation of the fermionic and bosonic partner of the pseudo NG boson, $\psi$ and $a$, is due to SUSY breaking and can be understood when considering the hidden sector alone, neglecting couplings to the visible sector. After breaking of SUSY, soft terms are induced in the scalar potential of the hidden sector. They have the form

$$
V_{\text {soft }}=m_{3 / 2}^{2}\left(|X|^{2}+\left|V_{12}\right|^{2}+\left|V_{34}\right|^{2}\right)+2 m_{3 / 2}\left(\Lambda^{2} X+\text { h.c. }\right),
$$


where we suppressed $\mathcal{O}(1)$ prefactors 10 The supersymmetric part of the scalar potential reads

$$
V_{\mathrm{SUSY}}=\left|V_{12} V_{34}-\Lambda^{2}\right|^{2}+\left|X V_{12}\right|^{2}+\left|X V_{34}\right|^{2}
$$

and (B.1) and (B.2) have together a minimum at 11

$$
\langle X\rangle \simeq-m_{3 / 2}\left(1+\frac{3}{2} \frac{m_{3 / 2}^{2}}{\Lambda^{2}}\right), \quad\left\langle V_{12}\right\rangle=\left\langle V_{34}\right\rangle \simeq \Lambda-\frac{m_{3 / 2}^{2}}{\Lambda} .
$$

The vev of $X$ induces a Dirac-mass term for the fermion partners of $V_{12}$ and $V_{34}$, which finally gives a mass close to $m_{3 / 2}$ to the fermionic partner of the pseudo NG boson, $\psi$. By expanding the fields like $V_{12}=\left\langle V_{12}\right\rangle+\rho / 2+\ldots$ and $V_{34}=\left\langle V_{34}\right\rangle-\rho / 2+\ldots$ one can furthermore derive that the mass of the bosonic partner of the pseudo NG boson is given by $m_{\rho} \simeq 4 m_{3 / 2}$. For definiteness, we use these masses in the phenomenological analysis, and assume that $\psi$ is somewhat heavier than the gravitino $\psi_{3 / 2}$, although the exact values can change depending on the details of the Kahler potential and SUSY breaking sector.

Without coupling to the visible sector, the pseudo NG boson in the hidden sector would remain massless after SUSY breaking. However, the coupling to the right-handed neutrinos generates an additional term $\left(f_{3}^{2} \Lambda^{2} / M_{3}\right) V_{34} V_{34}$ in the superpotential, which after SUSY breaking give rise to terms like $\left(f_{3}^{2} m_{3 / 2} \Lambda^{2} / M_{3}\right) V_{34} V_{34}+$ h.c. in the scalar potential. Noting that $V_{34}=\Lambda e^{-i a / 2 \Lambda}+\ldots$, it follows that the mass of the pseudo NG boson in the hidden sector is of the order of $m_{a} \sim f_{3} \sqrt{m_{3 / 2} \Lambda^{2} / M_{3}}$, see Eq. (2.9).

\section{References}

[1] W. Buchmuller, L. Covi, K. Hamaguchi, A. Ibarra and T. Yanagida, JHEP 0703 (2007) 037.

[2] S. Bobrovskyi, W. Buchmuller, J. Hajer and J. Schmidt, arXiv:1007.5007 [hep-ph].

[3] B. C. Allanach, A. Dedes and H. K. Dreiner, Phys. Rev. D 60 (1999) 075014.

[4] M. Chemtob, Prog. Part. Nucl. Phys. 54 (2005) 71.

[5] A. A. Abdo et al., Phys. Rev. Lett. 104 (2010) 091302.

[6] M. Fukugita and T. Yanagida, Phys. Lett. B 174 (1986) 45.

[7] T. Yanagida, in Proc. of the Workshop on "the Unified Theory and the Baryon Number in the Universe", Tsukuba, Japan, Feb. 13-14, 1979, eds. O. Sawada and S. Sugamoto, (KEK Report KEK-79-18, 1979, Tsukuba) p.95; Progr. Theor. Phys. 64 (1980) 1103; P. Ramond, in a Talk given at Sanibel Symposium, Palm Coast,

\footnotetext{
${ }^{10}$ There can also be terms of the form $m^{\prime} X\left(V_{12} V_{34}-\Lambda^{2}\right)$, with $m^{\prime} \sim m_{3 / 2}$, which we will neglect.

${ }^{11}$ Note, that one has to take into account additional terms of the form $|X|^{4} / \Lambda^{2}$ in the Kahler potential to understand the stability of the above minimum when SUSY is broken.
} 
Fla., Feb. 25-Mar. 2, 1979, preprint CALT-68-709. See also S. Glashow, in Proc. of the Cargése Summer Institute on "Quarks and Leptons", Cargése, July 9-29, 1979, eds. M. Lévy et. al, (Plenum, 1980, New York), p.707.

[8] For an early work, see P. Minkowski, Phys. Lett. B 67 (1977) 421; see also R. N. Mohapatra and G. Senjanovic, Phys. Rev. Lett. 44 (1980) 912; J. Schechter and J. W. F. Valle, Phys. Rev. D 22 (1980) 2227.

[9] S. Davidson and A. Ibarra, Phys. Lett. B 535 (2002) 25; W. Buchmuller, P. Di Bari and M. Plumacher, Annals Phys. 315 (2005) 305.

[10] M. Bolz, A. Brandenburg and W. Buchmüller, Nucl. Phys. B 606 (2001) 518;

J. Pradler and F. D. Steffen, Phys. Rev. D 75, 023509 (2007); V. S. Rychkov and A. Strumia, Phys. Rev. D 75, 075011 (2007).

[11] M. Kawasaki, K. Kohri and T. Moroi, Phys. Rev. D 71, 083502 (2005).

[12] K. Jedamzik, Phys. Rev. D 74 (2006) 103509.

[13] M. Pospelov, Phys. Rev. Lett. 98 (2007) 231301.

[14] K. Kohri and F. Takayama, Phys. Rev. D 76, 063507 (2007); M. Kaplinghat and A. Rajaraman, Phys. Rev. D 74, 103004 (2006); R. H. Cyburt et al. JCAP 0611, 014 (2006); K. Hamaguchi et al. Phys. Lett. B 650 (2007) 268; J. Pradler and F. D. Steffen, Phys. Lett. B 666, 181 (2008); M. Pospelov, J. Pradler and F. D. Steffen, JCAP 0811 (2008) 020.

[15] T. Kanzaki et al. Phys. Rev. D 75 (2007) 025011.

[16] J. L. Diaz-Cruz, J. R. Ellis, K. A. Olive and Y. Santoso, JHEP 0705 (2007) 003.

[17] M. Ratz, K. Schmidt-Hoberg and M. W. Winkler, JCAP 0810 (2008) 026.

[18] J. Pradler and F. D. Steffen, Phys. Lett. B 648 (2007) 224.

[19] W. Buchmuller, K. Hamaguchi, M. Ibe and T. T. Yanagida, Phys. Lett. B 643 (2006) 124 .

[20] L. Boubekeur, K. Y. Choi, R. R. de Austri and O. Vives, JCAP 1004 (2010) 005;

J. Pradler and F. D. Steffen, Nucl. Phys. B 809 (2009) 318.

[21] A. De Simone, M. Garny, A. Ibarra and C. Weniger, JCAP 1007 (2010) 017.

[22] C. Cheung, J. Mardon, Y. Nomura and J. Thaler, JHEP 1007 (2010) 035.

[23] S. W. Hawking, Phys. Lett. B 195 (1987) 337.

[24] S. R. Coleman and K. M. Lee, Nucl. Phys. B 329 (1990) 387. 
[25] K. Kurosawa, N. Maru and T. Yanagida, Phys. Lett. B 512 (2001) 203.

[26] W. Buchmuller and J. Schmidt, Nucl. Phys. B 807 (2009) 265.

[27] M. Kuriyama, H. Nakajima and T. Watari, Phys. Rev. D 79 (2009) 075002.

[28] V. Barger, P. Fileviez Perez and S. Spinner, Phys. Rev. Lett. 102 (2009) 181802;

P. Fileviez Perez and S. Spinner, Phys. Lett. B 673 (2009) 251.

[29] N. Seiberg, Phys. Rev. D 49 (1994) 6857.

[30] K. I. Izawa and T. Yanagida, Prog. Theor. Phys. 95 (1996) 829.

[31] H. K. Dreiner and G. G. Ross, Nucl. Phys. B 410 (1993) 188.

[32] H. K. Dreiner, arXiv:hep-ph/9707435.

[33] F. Takayama and M. Yamaguchi, Phys. Lett. B 485 (2000) 388.

[34] A. A. Abdo et al., Phys. Rev. Lett. 104 (2010) 091302.

[35] A. Arvanitaki, S. Dimopoulos, S. Dubovsky, P. W. Graham, R. Harnik and

S. Rajendran, Phys. Rev. D 79 (2009) 105022.

[36] L. J. Hall, K. Jedamzik, J. March-Russell and S. M. West, JHEP 1003, 080 (2010).

[37] E. W. Kolb and M. S. Turner, Nature 294, 521 (1981).

[38] K. Griest and M. Kamionkowski, Phys. Rev. Lett. 64, 615 (1990).

[39] J. L. Feng, H. Tu and H. B. Yu, JCAP 0810, 043 (2008).

[40] S. Asai, K. Hamaguchi and S. Shirai, Phys. Rev. Lett. 103 (2009) 141803; S. Asai, K. Hamaguchi and S. Shirai, Phys. Rev. Lett. 103 (2009) 141803.

[41] Y. B. Zeldovich, I. Y. Kobzarev and L. B. Okun, Zh. Eksp. Teor. Fiz. 67 (1974) 3 [Sov. Phys. JETP 40 (1974) 1].

[42] J. Preskill, S. P. Trivedi, F. Wilczek and M. B. Wise, Nucl. Phys. B 363, 207 (1991). 